gastritis may be associated with an ulcer crater on the pyloric ring or in the prepyloric stomach. It is cured by vagal section yet the disease is commonly associated with absent free hydrochloric acid in the fasting juice and a low response to histamine. In vagotomy we have, in a sense, the right operation found for the wrong reasons. We must, therefore, not let low acid findings deter us from using vagal section in the treatment of chronic peptic disease.-I am, etc.,

\section{HAROLD BURge.}

West London Hospital,

\section{REFERENCE} Butsch, W. L., Proceedings of the Staff Meetings
of the Mayo Clinic, 1935, 10, 435 .

\title{
Coagulation Failure in Pregnancy
}

SIR,-I have suggested (14 March, p. single experiment, but the above does seem 691) that amniotic fluid can be implicated to corroborate the circumstantial evidence as a cause of clinical coagulation fluid in available. ${ }^{3}$ While present in an in-vitro syspregnancy. It seems that amniotic failure does tem amniotic fluid increased the duration of not merely contain a potent coagulant but is contractions and diminished their frequency, in addition a highly active and variable sub- but when removed from the same system it stance. ${ }^{1}$

Uterine hypotonia appears to be a constant feature in cases of amniotic fluid embolism. Furthermore, Attwood and Landing $^{2}$ have found amniotic fluid in the veins of uteri removed for persistent postpartum haemorrhage, and occasionally it has been the only abnormal finding. ${ }^{3}$ If amniotic fluid does cause uterine hypotonia its effect could be central or local. If its effect is local one but when removed from the same system it
left the muscle in an atonic state. This could possibly indicate a dual effect, the dominant and more permanent one being that of inhibition. Could this be the basis of postpartum uterine hypotonia and also postpartum haemorrhage in those cases where no other case can be found?

It is interesting to note that numbered among the causes of postpartum uterine hypotonia are multiparity, multiple pregTracing' $B$
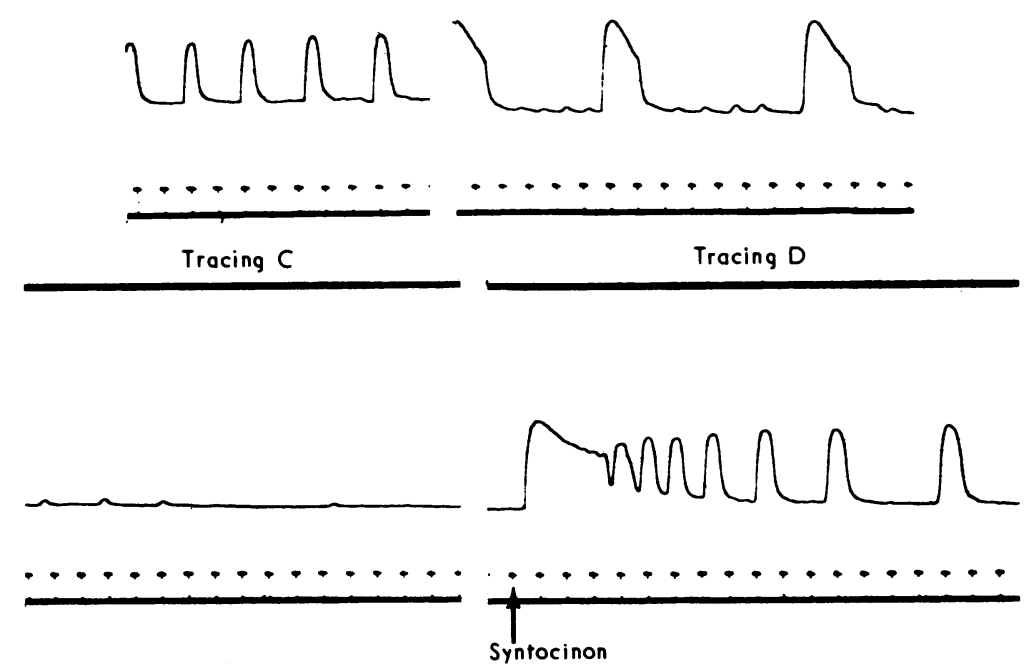

TRACING A.-Spontaneous uterine muscle contractions in vitro. TRACING B.-Effect of introduction of $5 \mathrm{ml}$. of amniotic fluid into the system. TRACING C. Obtained when introduced into the system.

could expect it to inhibit uterine muscle in vitro.

During an in-vitro study of uterine function amniotic fluid was added to a standard uterine muscle preparation." Tracing A shows spontaneous uterine muscle contractions. Tracing $B$ shows the effect of the introduction of $5 \mathrm{ml}$. of amniotic fluid into the system. It can be seen that, while the duration of the contractions increased, their frequency diminished from one in every two minutes to one in every six. Tracing $C$ was obtained when the amniotic fluid was washed out of the system. Spontaneous contractions did not return, and the muscle remained almost totally atonic until synthetic oxytocin (Syntocinon) was added (Tracing D).

Conclusions cannot be drawn from a nancy, hydramnios, placenta praevia, and precipitate labour. ${ }^{5}$ These conditions are also associated with fatal amniotic fluid embolism. The histological findings of Attwood and Landing may not be coincidental. The relevance of amniotic fluid seems to be worth further consideration.-I am, etc.,

\section{Louis D. Courtney.}

Radcliffe Infirmary Oxford.

REFERENCES

Courtney, L. D., and Allington, M., to be published.

Landing, B. H., New England fournal of Medicine $950,243,590$

3 Courtney, L. D., M.D. thesis. 1970. Submitted. Lewis, V. and Pybus, A., Unpublished observa-
tions, 1970 .

i Donald, I., Practical Obstetric Problems, 3rd edn

\section{Jet Vaccination}

SIR,-Your leading article on jet vaccination (10 October, p. 64) leads me to question the safety of this procedure. The injector is not sterilized between injections, and in many cases it produces a small amount of bleeding at the injection site. The injector could thus become contaminated with blood, thus transmitting, for instance, hepatis virus to subsequent patients.-I am, etc.,

Bracknell,
Berks.

M. G. KREMER.

SIR,-Your leading article on jet vaccination (10 October, p. 64) rightly deprecates the use of jet injection for primary vaccination with killed vaccines. But you miss one point which equally applies to booster doses, which you say can be given satisfactorily by jet injection. If all or part of the dose is injected subcutaneously, general reactions are likely to ensue, and the main advantage of intradermal injection is lost.-I am, etc.,

\section{Royal Free Hospital, North-W estern Bra
London N.W.3.}

W. P. STamm.

\section{Attitudes to Psychiatry}

SIR,-We were most interested to note the conclusions of a recent article "Attitudes to Psychiatry in the General Hospital" (10 October p. 106). However, like the authors, we have also wondered whether their methods led them to obtain a much rosier picture of the position of psychiatry than in fact really exists.

We base this comment not on speculation but on a preliminary examination of our own study of attitudes to psychiatry, which has just been completed. This study, which was designed and begun by one of us (G.P.M.) in 1967, has examined the change of attitudes to psychiatry within all the nonpsychiatric staff of a district general hospital over a period of three years. We have, in particular, been attempting to look at the effect of a purpose-built 100-bedded psychiatric unit and day hospital on such attitudes. The unit has since 1966 served an urban and rural catchment population along the lines advocated in the 1962 Hospital Plan for England and Wales.

Our results, which we hope to report fully in the near future, indicate that, while some favourable attitude change has occurred, a great deal of uncertainty and prejudice remains. For example, there has been no significant change in the proportion of the sisters and administrative staff, who clearly agree they have "much less patience" with the mentally ill $(40 \%)$, view the mentally ill as having "much less sense of responsibility" (50\%), consider them "uncooperative and difficult" as patients $(23 \%)$, and who advocate sterilization for severe mental illness $(39 \%)$.

Two quite important differences in method may be responsible for the less favourable views we have obtained. Firstly, we attempted to preserve the anonymity of 
our respondents. Secondly, we based our 50 -item questionnaire on verbatim attitude and opinion statements derived from interviews with members of the various hospital staff groups under study.

While we would agree that it is important to stress the positiveness of attitudes to psychiatry, we consider it equally if not more important to define the areas of prejudice that still exist and which at least up to date defy change. For such prejudice might seriously hamper the further integration of psychiatry in the general hospital and yet go undetected. The fuller analysis of our findings should help to clarify what attitudes still require change and hence lead to a more rational teaching programme for all general hospital staff. Finally, we would hope our results will encourage research into methods of teaching by which such prejudices can be overcome.-We are, etc.,

\section{G. P. Maguire.}

Department of Psychiatry,

\section{F. McEvetr.}

\section{Department of Psychiatry, \\ Queen Elizabeth II Hospital,}

Welwyn Garden City, Herts.

\section{Renin Activity in Acute Renal Failure}

SIR,-Dr. J. J. Brown and others (31 January, p. 253) have recently reported a significant increase in the renin concentration of peripheral venous blood in most of their patients with acute renal failure. The highest levels were found during the first two weeks after onset, while lower levels were detected during the diuretic phase.

We wish to record our experience with nine patients (five men and four women) with acute renal failure of diverse aetiology: surgery on the gastrointestinal or biliary tract, four; eclampsia, two; and crush injury, heat exhaustion with hypotension, and acute gouty nephropathy, one case each. Acute tubular necrosis was proved by kidney biopsy in four patients. Renin

Renin and Renin Activity in Acute Renal Failure

\begin{tabular}{|c|c|c|c|}
\hline Authors & 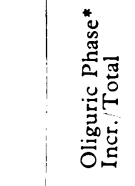 & 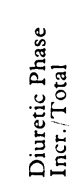 & 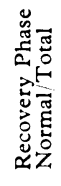 \\
\hline $\begin{array}{l}\text { Tu } \\
\text { Kokot and Kuska } \\
\text { Brown et al. } \\
\text { This Report }\end{array}$ & $\begin{array}{c}8 / 18 \\
28 / 33 \\
22 / 24 \\
4 / 5\end{array}$ & $\begin{array}{l}1 / 8 \\
8 / 19 \\
4 / 8 \\
5 / 6\end{array}$ & $\begin{array}{l}1 / 1 \\
1 / 1\end{array}$ \\
\hline $\begin{array}{l}\text { Total } \ldots \\
\% \text { Increase (mean) }\end{array}$ & \begin{tabular}{l|l}
$\ldots$ & $62 / 80$ \\
$\ldots$ & 77.5
\end{tabular} & $\begin{array}{c}18 / 41 \\
44\end{array}$ & $2 / 2$ \\
\hline
\end{tabular}

*Daily urine volume less than $1,000 \mathrm{ml}$.

activity of peripheral venous blood, measured by the procedure of Pickens et $a l .{ }^{1}$ was increased above normal (normal range with subjects supine: $0 \cdot 1$ to $1.7 \mathrm{ng}$. of angiotensin II per hour per one $\mathrm{ml}$. of serum) on one or more occasions in seven patients. Renin activity ranged from $2 \cdot 0$ to $8.9 \mathrm{ng} . / \mathrm{ml}$. in four of the five patients studied during the oliguric phase, and from 2.0 to $12.2 \mathrm{ng} . / \mathrm{ml}$. in five of the six patients observed during the diuretic phase. There was no correlation between the levels of renin activity and serum sodium, potassium, systolic, diastolic, and mean blood pressure. Our findings are summarized in the Table along with results reported by other investigators.

Although the clinical material summarized above included a variety of aetiologies, and the methods of assay employed were dissimilar, it is apparent that during the oliguric phase increased levels of renin or renin activity were found in most instances. We, as others, found no correlation between the levels of renin activity or renin and of serum sodium, potassium, urea, or creatinine. As regards blood pressure, $\mathrm{Tu}^{2}$ found a slight positive correlation with renin activity, Dr. Brown and colleagues found a negative correlation, while we and Kokot and Kuska ${ }^{3}$ observed no correlation. These findings strongly suggest that the stimulus for the increase in renin secretion in acute renal failure arises within the kidney itself, rather than extrarenally.

The possible pathogenetic significance of increased renin in acute renal failure has been exhaustively discussed by Dr. Brown and his colleagues. We believe that their provocative interpretation has considerable merit. Recent studies by Hollenberg et al. ${ }^{4}$ have shown that in acute renal failure precipitated by nephrotoxic agents as in that caused by other aetiologies, the outstanding feature is marked reduction in cortical blood flow, with decrease in filtration pressure and filtration rate. ${ }^{5}$ These observations lend support to the concept that in the pathogenesis of acute renal failure activation of a vasomotor intrarenal pathway plays a primary role. In 1945 Goormaghtigh ${ }^{6}$ drew attention to the prominence of the juxtaglomerular apparatus in the crush syndrome and suggested that excessive release of renin and production of angiotensin might be responsible for the spasm of the glomerular afferent arteriole leading to reduction in glomerular filtration rate. To date evidence in support of or against this hypothesis is still lacking. If the increase in the blood levels of renin or renin activity found in clinical and experimental acute renal failure is coincidental or simply the result of the intrarenal disturbance, then the cortical ischaemia of acute renal failure is due to other vasoconstrictor substances. Alternatively, although in acute renal failure renin is markedly increased, lack of adequate amounts of substrate and/or of converting enzyme prevents the formation of angiotensin II within the kidney. Another possibility might be that angiotensin II is inactivated as soon as it is generated.

Oparil et al. ${ }^{7}$ have found that direct infusion of angiotensin I into the lung of the anaesthetized dog resulted in $54 \%$ conversion to angiotensin II. By contrast, infusion of angiotensin $I$ into the renal artery yielded only 7 to $9 \%$ conversion. Whether these experimental findings are applicable to clinical renal failure in man remains to be seen. None the less, even assuming that the kidney has a smaller capacity for converting angiotensin I to angiotensin II, under the appropriate circumstances the angiotensin II produced may well have pathogenetic importance, as Dr. Brown and his colleagues have suggested.-We are, etc.,

F. DEL GRECO. F. KRUMLOVSKY.

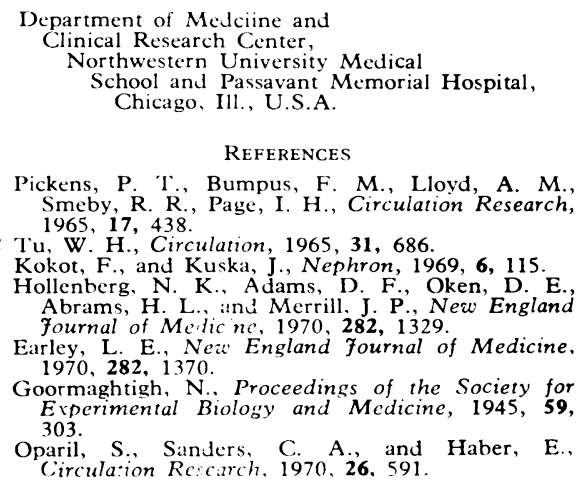

Pickens, P. T., Bumpus, F. M., Lloyd, A. M., Smeby, R. R., Page, I. H., Circularion
$1965,17,438$.

Tuu W. H., Circulation, 1965, 31, 686.

Hollenberg, N. K., Adams, D. F., Oken, D. E Abrams, H. L. ind Merrill, J. P., New England formal of Metic ne. $1970, \mathbf{2 8 2}, 1329$.

Earley, L. E., New England fournal of Medicine.

$1970,282,1370$.

Experimental Biology and Medicine, 1945, 59,
Eormaghtigh, 303.

Oparil, S., Sanders, C. A., and Haber, E.,
Circulation Recarch. 1970, 26, 591 .

\section{Treatment of Anal Fissure}

SIR,-I am currently conducting a fouryear follow-up of lateral subcutaneous internal sphincterotomy for chronic anal fissure in 98 patients in Colchester, and I am pleased to say that the findings are in accord with those of $\mathrm{Mr}$. D. C. Hoffmann and Professor J. C. Goligher (19 September, p. 673). There appears to be a slightly higher incidence of haematoma formation, but there has been only one case admitting to minor defects of anal continence. There have been no cases of recurrent fissure, and in only one patient was the sphincterotomy slightly enlarged at a later date when a prolapsing pile was excised.

I believe that this treatment for chronic fissure is the most satisfactory, particularly because anal continence is so well preserved, and in my series I have performed the operation for chronic fissure in three cases of ulcerative colitis. These patients are fully continent, whereas I would have suspected a degree of incontinence if sphincter stretching or posterior sphincterotomy had been performed.-I am, etc.,

\section{Lexden, Colchester. \\ Osteoporosis and Fluoride Therapy}

SIR,-Your interesting leading article (19 September, p. 660) indicates that there is only scanty information, particularly in the British literature, about the long-continued use of sodium fluoride in the management of osteoporosis in man. Because of this, the leading article quite properly advocates a cautious approach to fluoride therapy. In the context of possible long-term toxicity we should like to record our own experience with fluoride.

We began using sodium fluoride in 1962 and have so far treated 25 patients with osteoporosis, as well as 20 patients with Paget's disease. The results during the first five years were communicated to the Liverpool Medical Institution in 1967. Our 